\title{
Einfluss der Werkzeuggeometrie auf die Wärmeübertragung in Conchen
}

\author{
Jewe Schröder*, Peter Braun, Rolf Lämmli-Bürgler und \\ Heike P. Schuchmann
}

In memoriam H. G. Kessler (1932 - 1998)

Eine kontrollierte Temperaturführung ist für die Endveredelung der Schokolade entscheidend. Hierfür ist die Verbesserung der Wärmeübertragung im Conchierprozess maßgeblich. In dieser Arbeit werden Strömungssimulationen vorgestellt, die zeigen, dass die Wärmeübertragung durch Staueffekte am Werkzeug mehr begünstigt wird als durch direkten Abtrag. Experimentelle Untersuchungen bestätigten dies.

Schlagwörter: Conchierprozess, Schokolade, Temperaturführung, Wärmeübertragung

Eingegangen: 14. April 2008; akzeptiert: 13. Mai 2008

\section{Problemstellung}

Beim Conchierprozess handelt es sich um den abschließenden Veredelungsschritt der Schokoladenherstellung. Hierbei wird die $\mathrm{zu}$ Beginn pulverförmige Masse durch mechanischen Leistungseintrag in eine hochkonzentrierte Suspension umgewandelt [1]. Der Conchierprozess kann dabei in drei Phasen eingeteilt werden:

1. Befüllen (trockene Phase) - Mischen: Verringerung des Anteils an Wasser, Säuren und Aldehyden sowie Homogenisierung der Masse

2. Conchieren (pastöse Phase) - Hohe Scherung: Desagglomerieren der im Walzwerk entstandenen Agglomerate und Homogenisierung der dispers verteilten Zucker- und Kakaopartikel im frei werdenden Fett (Versalbung der Masse)

3. Verflüssigen (flüssige Phase) - Intensives Mischen: Verflüssigung nach Zugabe von Kakaobutter und Lecithin durch Einstellen einer multidispersen Suspensionsstruktur und weiteres Homogenisieren

Durch das Aufschmelzen des Fetts und das Freisetzen des in den Agglomeraten gebundenen Fetts bildet sich eine pastöse Masse mit zunehmender Viskosität und Fließgrenze. Sobald alle Einzelpartikel umhüllt sind und freies Fett vorliegt, sinken die Werte rapide ab. In der pastösen Phase ist der mechanische Leistungseintrag aufgrund der angestiegenen Viskosität am höchsten [1]. Durch Reibungsverluste erhöht sich die Temperatur in der Masse. In dieser Prozessphase diffundieren die Aromastoffe von den Kakaopartikeln durch das Fett auf die Zuckerpartikel. Auch hierfür ist neben dem Gesamtenergieeintrag die Höhe des Leistungseintrags entscheidend [2]. Die korrekte Temperaturführung spielt eine essentielle Rolle für die Qualität der bearbeiteten Schokoladenmasse, da alle physikalisch-chemischen Vorgänge ebenso wie die Aromabildung während des Conchierens temperaturabhängig sind $[1,3]$. Zur kontrollierten Temperaturführung muss die thermische Energie effizient abgeführt werden, wozu üblicherweise der Mantel der Conche extern mit Wasser temperiert wird. Für den Wärmestrom ist neben der Übertragungsfläche $A$ und dem treibenden Temperaturgefälle $\Delta T$ der Wärmedurchgangskoeffizient $k$ von Bedeutung, s. Gl. (1). Dieser wird durch die Wandstärke des Mantels $s$, die jeweiligen Wärmeleitfähigkeiten $\lambda$ sowie die strömungsabhängigen inneren und äußeren Wärmeübertragungskoeffizienten $\alpha_{\mathrm{i}}$ und $\alpha_{\mathrm{a}}$ bestimmt, s. Gl. (2) [4].

$\dot{Q}=k A \Delta T$

$k=\frac{1}{a_{\mathrm{i}}}+\frac{s}{\lambda}+\frac{1}{a_{\mathrm{a}}}$

Die Conchierwerkzeuge drehen während des Conchierens in unterschiedlichen Richtungen. Abb. 1 zeigt, dass je nach Drehrichtung die Masse verstärkt geschert oder die

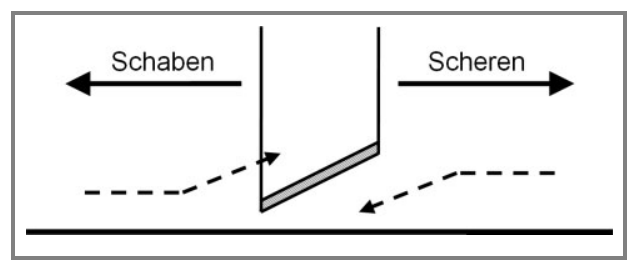

Abbildung 1. Schematische Darstellung der Funktionsweise der Conchierwerkzeuge in Abhängigkeit der Drehrichtung.

\section{Beim Conchierpro- zess handelt es sich um den abschlie- Benden Verede- lungsschritt der Schokoladenherstel- lung. Die korrekte Temperaturführung spielt eine essen- tielle Rolle für die Qualität der bear- beiteten Schokola- denmasse, da alle physikalisch-chemi- schen Vorgänge ebenso wie die Aro- mabildung dabei temperaturabhän- gig sind.}




\section{Als Maß für den Wärmestrom zwi- schen Schokoladen- masse und Wasser- kreislauf wurde der relative Wärme- durchgangskoeffi- zient herangezo- gen. Experimentelle Untersuchungen zeigen die Abhän- gigkeit von der rela- tiven Drehzahl und -richtung.}

Masse von der Kesselwand abgetragen wird [1]. Gezielte Untersuchungen zum Einfluss der Werkzeuggeometrie und des Abstands zwischen Werkzeug und Kesselwand auf die lokalen Strömungsverhältnisse sowie auf den Leistungseintrag und die Wärmeübertragung sind bislang nicht veröffentlicht worden. Anhand des Wärmedurchgangskoeffizienten $k$ wurde der Einfluss dieser Größen auf die Wärmeübertragung untersucht. Zur Erklärung der Messergebnisse erfolgte die Simulation der lokalen Strömungsverhältnisse um die Werkzeuge.

\section{Experimentelle Untersuchungen}

Für die experimentellen Arbeiten wurde eine horizontale Einwellenconche verwendet. Eingesetzt wurden eine Standard- und alternative Werkzeugmodifikationen. In der Standardmodifikation waren auf der Welle zwölf Conchierwerkzeuge so angebracht, dass jedes Volumenelement der Masse bei einer Umdrehung zweimal mit einem Werkzeug in Interaktion trat. Der Spalt zwischen Werkzeug und Kesselwand betrug an der engsten Stelle $2 \mathrm{~mm}$. Zum Vergleich wurden verschiedene Modifikationen vorgenommen:

1. Die Spaltweite zwischen Werkzeug und Kesselwand wurde zwischen 2 und $10 \mathrm{~mm}$ variiert.

2. Bei $10 \mathrm{~mm}$ Spaltweite waren sechs zusätzliche Werkzeuge mit einer Spaltweite von $2 \mathrm{~mm}$ montiert, die in beide Drehrichtungen einen schabenden Effekt besitzen und bei einer Umdrehung die gesamte Kesselwand einmal abschaben. Bei scherender Fahrweise wird somit zeitgleich geschert und die Kesselwand abgeschabt.

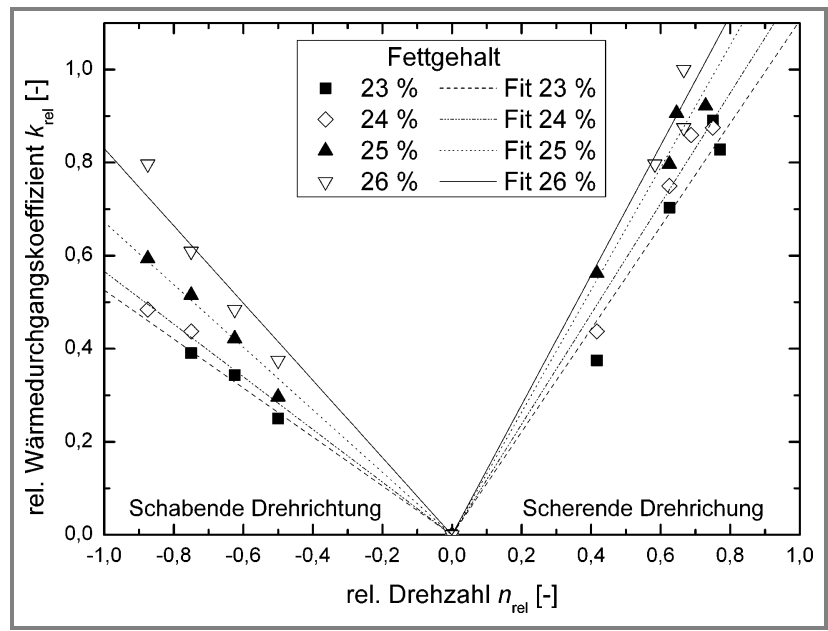

Abbildung 2. Abhängigkeit des relativen Wärmedurchgangskoeffizienten vom Fettgehalt, der relativen Drehzahl und der Drehrichtung (Standardmodifikation).
Die Kesselwand war zu mehr als der Hälfte von einem wassergefüllten Doppelmantel umgeben, der zur Temperierung der Schokoladenmasse diente.

Als Maß für den Wärmestrom zwischen Schokoladenmasse und Wasserkreislauf wurde der relative Wärmedurchgangskoeffizient $k_{\text {rel }}$ herangezogen, wobei sich alle Werte auf den maximalen gemessenen Wärmedurchgangskoeffizienten bei einer Spaltweite von $2 \mathrm{~mm}$ bezogen, s. Gl. (3).

$k_{\text {rel }}=k / k_{\max , 2 \mathrm{~mm}}$

Zur Berechnung wurde ein quasistationärer Zustand angenommen, in dem nur Leistung über die Werkzeuge eingebracht wird und ein definierter Wärmestrom von der Masse in den Wasserkreislauf abfließt. Dabei bleibt die Temperatur der Masse selbst konstant. Um diesen Randbedingungen $\mathrm{zu}$ entsprechen und Wärmeverluste zu minimieren, wurde der Kessel soweit wie möglich isoliert und mit temperierter Luft belüftet. Unter diesen Randbedingungen ließ sich der Wärmedurchgangskoeffizient aus der effektiven mechanischen Leistung unter Berücksichtigung der Verluste in Motor, Getriebe und Lagerung - sowie der Temperaturdifferenz zwischen Masse und Wasser ermitteln.

Die Aufgabemasse bestand aus $41 \%$ Kakaomasse und $59 \%$ Zucker und wies damit einen Fettgehalt von $22 \%$ auf. Die Primärpartikelgröße des Pulvers wurde durch vorheriges Mahlen einheitlich auf unter $19 \mu \mathrm{m}$ eingestellt. Um den Einfluss der Strukturveränderung gezielt zu untersuchen, wurde während des Conchiervorgangs der Fettgehalt durch gezielte Fettzugaben stufenweise bis auf $27 \%$ erhöht. Oberhalb von 27 \% begann die Verflüssigung.

Abb. 2 zeigt die Abhängigkeit des relativen Wärmedurchgangskoeffizienten $k_{\text {rel }}$ von der relativen Drehzahl, s. Gl. (4), und der Drehrichtung des Conchierwerkzeugs für ausgewählte Fettgehalte der Masse.

$n_{\text {rel }}=n / n_{\max }$

Wie erwartet verbessert sich mit steigender Drehzahl in beiden Drehrichtungen der Koeffizient aufgrund des höheren Stoffaustauschs. Des Weiteren wächst er mit steigendem Fettgehalt und der damit ablaufenden Strukturänderung der Masse. Dies kann durch die bessere Wärmeleitung in der homogeneren Masse und durch den Übergang vom pulverförmigen in den pastösen kontinuierlichen Zustand begründet werden. In scherender Fahrweise ist der Wärmedurchgangskoeffizient im Vergleich 
zu schabender Fahrweise höher, was entgegen der Erwartung steht, dass durch den Abtrag der Masse an der Kesselwand eher eine Verbesserung des Wärmestroms zu erwarten ist. Verantwortlich für dieses Phänomen ist wahrscheinlich der in den anschließenden Simulationen beobachtete Staueffekt.

In Abb. 3 ist der Verlauf des relativen Wärmedurchgangskoeffizienten $k_{\text {rel }}$ mit steigendem Fettgehalt und damit ablaufender Strukturänderung in der Masse bei zwei Spaltweiten dargestellt. Mit aufgetragen sind die Werte, die mit der modifizierten Geometrie (beidseitige Schabeelemente) erhalten wurden.

Deutlich erkennbar ist, dass der Koeffizient ohne Modifikation der Werkzeuge (geschlossene Symbole) bei einer Spaltweite von $10 \mathrm{~mm}$ immer sehr viel niedriger liegt als bei $2 \mathrm{~mm}$. Während er bei einer Spaltweite von $2 \mathrm{~mm}$ weitgehend unabhängig vom Fettgehalt der Masse ist, fällt er bei $10 \mathrm{~mm}$ ab einem Fettgehalt von $26 \%$ deutlich ab, obwohl in diesem Bereich ein hoher mechanischer Leistungseintrag stattfindet und somit eine gute Wärmeabfuhr nötig ist. Hingegen ist beim Einsatz der zusätzlichen Schaber (offenes Symbol) genau in diesem Bereich eine klare Steigerung von $k_{\text {rel }}$ erkennbar, was sich auf den verbesserten Stoffaustausch zurückführen lässt.

Bei allen Werkzeugmodifikationen bzw. Abständen konnte der gleiche maximale mechanische Leistungseintrag erreicht werden. Die Partikelgrößenverteilungen, der Wassergehalt und die Fließkurven der hergestellten Massen zeigten keine relevanten Abweichungen.

\section{Strömungssimulation}

Um die experimentellen Ergebnisse besser interpretieren zu können, wurden die lokalen Strömungsverhältnisse in unmittelbarer Umgebung des Werkzeugs und der Kesselwand mit Hilfe von ANSYS CFX vereinfacht simuliert. Dazu wurde ein 2D-Modell um ein Werkzeug in scherender Drehrichtung erstellt. Im Gegensatz zum realen System ruht das Werkzeug relativ zur Wand und die Masse fließt, weshalb an der Wand keine Haftung und am Werkzeug Haftung angenommen wurde. Variiert wurden die Anströmgeschwindigkeit der Masse, der Anstellwinkel der Schaber und die Breite des Spaltes zwischen Werkzeug und Wand. Während des Conchierprozesses verändern sich die Struktur und damit die rheologischen Eigenschaften der Masse. Physikalischchemische Einflussgrößen sind bereits untersucht, ein einheitliches rheologisches Modell für den gesamten Conchierprozess liegt allerdings bislang nicht vor [5]. Für diese orientie-

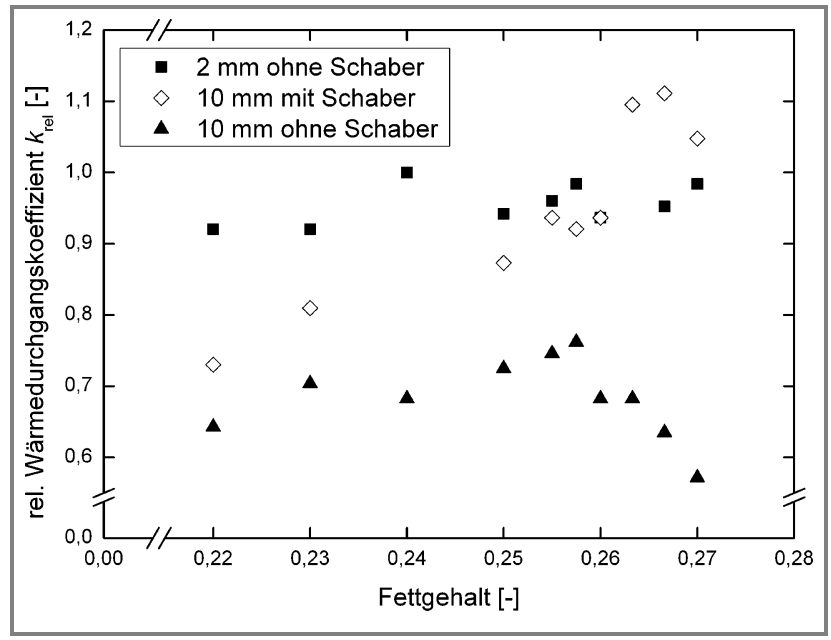

Abbildung 3. Abhängigkeit des relativen Wärmedurchgangskoeffizienten vom Fettgehalt (verschiedene Werkzeugmodifikationen, scherender Drehrichtung).

renden Simulationsrechnungen wurde daher das Modell von Herschel-Bulkley gewählt, s. Gl. (5) [6]. Der Wandel der Struktur wurde hierbei ausschließlich über die Veränderung der Fließgrenze $\tau_{0}$ im Bereich von $10 \mathrm{~Pa}$ bis 100.000 Pa beschrieben:

$\tau=\tau_{0}+k_{\mathrm{HB}} \dot{\gamma}^{\mathrm{n}_{\mathrm{HB}}}$

Bei Anstellwinkeln von $15^{\circ}$ und $30^{\circ}$ und einer Spaltweite von $5 \mathrm{~mm}$ wurde unabhängig von der Fließgrenze der Masse keine Förderung durch den konischen Spalt beobachtet. Der gesamte Volumenstrom wird über das Werkzeug umgelenkt. Mit steigender Spaltweite $(10,15$ und $30 \mathrm{~mm})$ war oberhalb einer Fließgrenze von 1.000 Pa eine deutliche Erhöhung der Geschwindigkeiten im Spalt erkennbar. Bei einer Spaltweite von $30 \mathrm{~mm}$ wurden mit bis $\mathrm{zu} 3,0 \mathrm{~m} / \mathrm{s}$ Geschwindigkeiten oberhalb der Anströmgeschwindigkeit festgestellt. Durch die mehr oder weniger stark ausgeprägten Staueffekte zwischen Werkzeug und Kesselwand anstatt des erwarteten Einzugs stieg die Strömungsgeschwindigkeit über dem Werkzeug auf ein Vielfaches der Anströmgeschwindigkeit an.

Der Anstellwinkel $\left(15^{\circ}\right.$ und $\left.30^{\circ}\right)$ beeinflusste die Größe der vertikalen Einzugszone bei gleicher Spaltweite. Bei niedriger Fließgrenze („verflüssigte Masse“) wurden bei einer großen Einzugszone höhere Geschwindigkeiten im Spalt berechnet. Mit steigender Fließgrenze („pastöse Masse“) kehrte sich dies um, was auf einen verstärkten Staueffekt durch die größere Einzugszone hinweist. Beim Absenken der Anströmgeschwindigkeit von 2,5 auf 2,0 bzw. $1,0 \mathrm{~m} / \mathrm{s}$ bei konstanter Geometrie ging der zuvor beobachtete exponentielle Anstieg der Ge-
Der Anstellwinkel beeinflusst die GröBe der vertikalen Einzugszone bei gleicher Spaltweite. Bei niedriger Fließgrenze wurden bei einer großen Einzugszone höhere Geschwindigkeiten im Spalt berechnet. Mit steigender Fließgrenze kehrte sich dies um, was auf einen verstärkten Staueffekt durch die größere Einzugszone hinweist. 


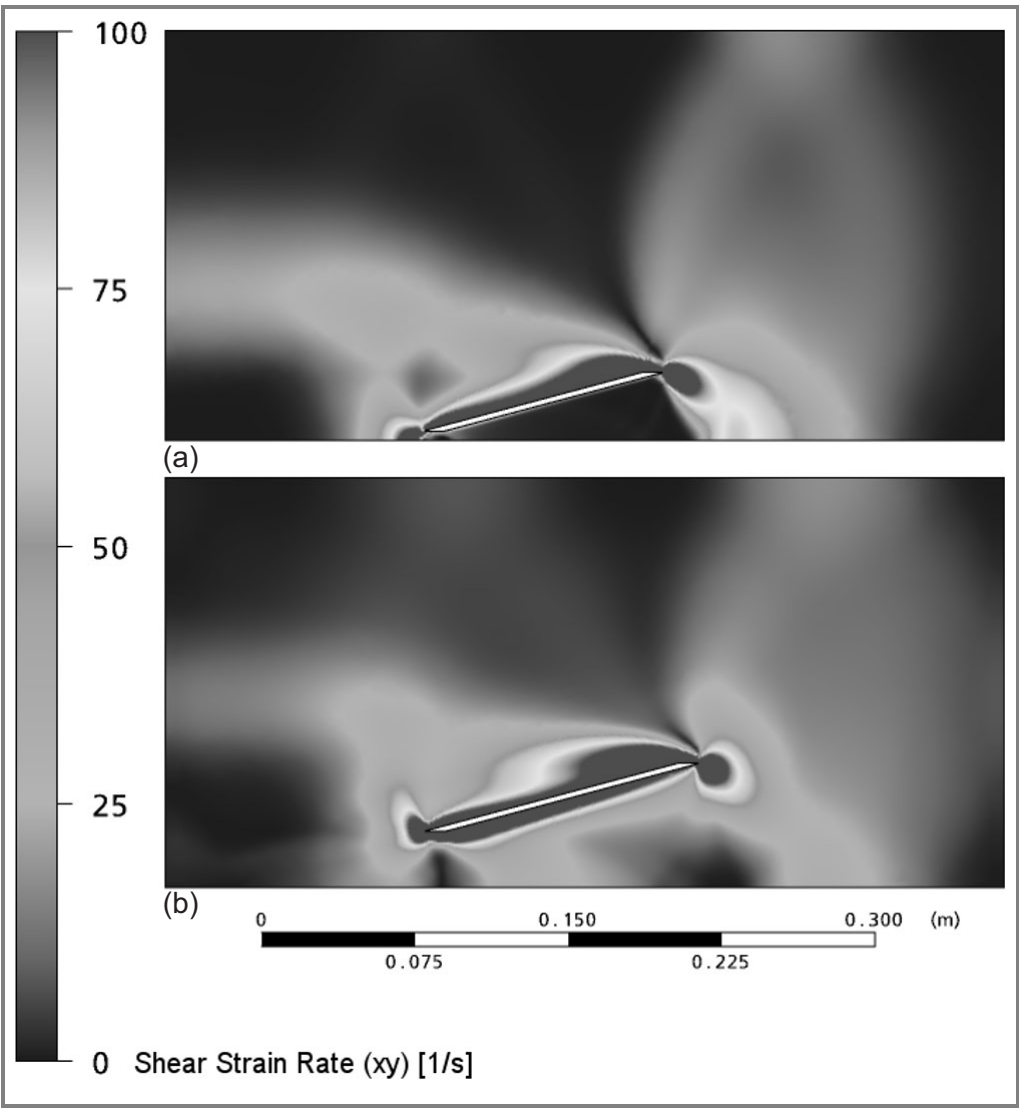

Abbildung 4. Vergleich der Scherrate bei Spaltweiten von (a) $5 \mathrm{~mm}$ und (b) $30 \mathrm{~mm}$ und konst. Randbedingungen (Fließgrenze $10.000 \mathrm{~Pa}$, Anströmgeschwindigkeit $2,5 \mathrm{~m} / \mathrm{s}$, Winkel $15^{\circ}$ )

\section{Schlussfolgerungen}

Die Untersuchungen zeigten die Bedeutung der Geometrie der Conchenwerkzeuge auf den Wärmeübergang. Geringe Abstände bzw. kleine Spaltweiten ermöglichen auch in scherender Fahrweise eine effiziente Wärmeabfuhr. Gründe hierfür liegen in den veränderten lokalen Strömungsverhältnissen. Die begleitende Strömungssimulation zeigte in scherender Fahrweise vor allem bei geringen Spaltweiten einen deutlichen Staueffekt, was zu einer Umlenkung der Strömung über das Werkzeug und Erhöhung der dort vorliegenden Strömungsgeschwindigkeiten führt und damit den Stoff- und Wärmeaustausch verbessert. Dies erklärt auch den in scherender Fahrweise gemessenen höheren Wärmedurchgangskoeffizienten. Über den Anstellwinkel des Werkzeugs lässt sich dieser Staueffekt gezielt für verschiedene rheologische Eigenschaften einstellen. Festgestellt wurde des Weiteren eine verstärkte Scherung der Masse oberhalb des Werkzeugs, die möglicherweise einen entscheidenden Einfluss auf die rheologischen Eigenschaften des Endprodukts hat. Der Einsatz von zusätzlichen Schabern ermöglicht eine signifikante Verbesserung des Wärmestroms während der entscheidenden Phase des hohen mechanischen Leistungseintrags.

\section{Dipl.-Ing. J. Schröder}

(jewe.schroeder@lvt.uni-karlsruhe.de), Universität Karlsruhe (TH), Institut für Biound Lebensmitteltechnik, Bereich I: Lebensmittelverfahrenstechnik, Kaiserstraße 12, D-76131 Karlsruhe, Germany;

Dr. P. Braun,

Dipl. Masch.-Ing. R. Lämmli-Bürgler, Bühler AG, CH-9240 Uzwil, Switzerland;

Prof. Dr.-Ing. H. P. Schuchmann,

Universität Karlsruhe (TH), Institut für Biound Lebensmitteltechnik, Bereich I:

Lebensmittelverfahrenstechnik, Kaiserstraße 12,

D-76131 Karlsruhe, Germany.

\section{Formelzeichen} Schüttguts noch der konzentrierten Suspension korrekt berücksichtigt. Die festgestellten Tendenzen sind dennoch übertragbar. Durch die nicht berücksichtigte Haftung an der Kesselwand sind im realen System verstärkt Scherung und Staueffekte zwischen Werkzeug und Kesselwand $\mathrm{zu}$ erwarten. Die Rundung des Kessels verursacht ein nicht konstantes Anströmprofil, was eine Abschwächung der Scherung oberhalb des Werkzeugs verursachen kann, falls der zuvor erwähnte verstärkte Staueffekt dies nicht ausgleicht.

\begin{tabular}{|c|c|c|}
\hline A & {$\left[\mathrm{m}^{2}\right]$} & Fläche \\
\hline$a$ & {$\left[\mathrm{~W} / \mathrm{m}^{2} \mathrm{~K}\right]$} & $\begin{array}{l}\text { Wärmeübertragungskoeffi- } \\
\text { zient }\end{array}$ \\
\hline & {$[1 / \mathrm{s}]$} & Scherrate \\
\hline & {$\left[\mathrm{W} / \mathrm{m}^{2} \mathrm{~K}\right]$} & $\begin{array}{l}\text { Wärmedurchgangskoeffi- } \\
\text { zient }\end{array}$ \\
\hline 3 & {$\left[\mathrm{~Pa} \mathrm{~s}^{\mathrm{n}}\right]$} & Herschel-Bulkley-Viskosität \\
\hline & {$[\mathrm{W} / \mathrm{m} \mathrm{K}]$} & Wärmeleitfähigkeit \\
\hline & {$[-]$} & Herschel-Bulkley-Exponent \\
\hline & {$[1 / \mathrm{m}]$} & Drehzahl \\
\hline
\end{tabular}




$\begin{array}{lll}\dot{Q} & {[\mathrm{~W}]} & \text { Wärmestrom } \\ s & {[\mathrm{~m}]} & \text { Dicke } \\ T & {[\mathrm{~K}]} & \text { Temperatur } \\ \tau & {[\mathrm{Pa}]} & \text { Schubspannung } \\ \tau_{0} & {[\mathrm{~Pa}]} & \text { Fließgrenze }\end{array}$

\section{Literatur}

[1] S. T. Beckett, Moderne Schokoladentechnologie, Behr's Verlag, Hamburg 1990.
[2] G. Ziegleder et al., Suesswaren, 2005, 1-2, 10.

[3] R. A. Martin, Adv. Food Res., 1987, 31, 211.

[4] VDI-Wärmeatlas, 9. Auflage, Springer-Verlag, Heidelberg 2002.

[5] P. Braun, Dissertation, ETH Zürich 2000.

[6] D. Weipert, H.-D. Tscheuschner, E. Windhab, Rheologie der Lebensmittel, Behr's Verlag, Hamburg 1993. 